\title{
Quantification of an oval optic disc in relation to myopic foveoschisis using swept-source optical coherence tomography
}

Ke Zhu ${ }^{1,2,3}$, Boya Lei ${ }^{1,2,3}$, Keyan Wang ${ }^{1,2,3}$, Fang Song ${ }^{1}$, Rui Jiang ${ }^{1,2,3}$, Qing Chang ${ }^{1,2,3}$, Gezhi X 1,2,3 $^{1,2}$ and Han Chen ${ }^{1,2,3^{*}}$

\begin{abstract}
Background: To investigate the relationship between an oval optic disc and the occurrence of myopic foveoschisis (MF) using swept-source optic coherence tomography (SS-OCT).

Methods: Fifty eyes of 25 patients with unilateral MF were included in this retrospective observational study. The biometric features of the optic disc and peripapillary structures were evaluated using SS-OCT.

Results: The ovality index $(\mathrm{OI})$ of the optic disc was significantly smaller $(P=0.003)$ and the optic disc tilt angle was greater $(P=0.023)$ in the eyes with MF than in the contralateral eyes. The optic disc tilt angle was significantly correlated with the OI $(P=0.000)$. Generalized estimating equation (GEE) model (linear regression) demonstrated that spherical equivalent refraction $(P=0.001)$, narrow macular staphyloma $(P=0.001)$ and the occurrence of MF $(P=0.026)$ were the independent factors associated with the Ol. Narrow macular staphyloma was more frequent $(P=0.020)$ and the staphyloma was deeper $(P=0.006)$ in eyes with MF. GEE model (logistic regression) revealed that narrow macular staphyloma was the only independent factor related to the occurrence of MF $(P=0.013)$.

Conclusions: An oval optic disc in eyes with MF resulted from the increased tilt around the vertical disc axis. The optic disc tilt was related to narrow macular staphyloma, which was the only independent factor associated with the occurrence of MF. The clinical relevance needs further exploration through longitudinal analysis.
\end{abstract}

Keywords: Myopic foveoschisis, Oval optic disc, Swept-source optic coherence tomography, Optic disc tilt, Narrow macular staphyloma

\section{Background}

High myopia is one of the most common causes of irreversible vision loss and blindness [1-3]. It has been estimated that high myopia will affect more than 1 billion people worldwide by $2050[4,5]$. The typical degeneration that occurs in highly myopic eyes increases the risk of macular diseases, including choroidal neovascularization, foveoschisis, and macular hole $(\mathrm{MH})[2,3,6,7]$.

*Correspondence: hancheneent@163.com

1 Eye Institute and Department of Ophthalmology, Eye and ENT Hospital, Fudan University, 83 Fen Yang Road, Shanghai 200031, China

Full list of author information is available at the end of the article
Myopic foveoschisis (MF), defined as splitting of the neurosensory retina, was first reported by Takano and Kishi in 1999 [8]. MF occurs in 8-34\% of highly myopic eyes [9], and may gradually develop to foveal detachment, $\mathrm{MH}$, or $\mathrm{MH}$-associated retinal detachment $[2,8]$. Although the pathogenesis of MF is still unclear, recent studies suggest that tangential traction from the posterior vitreous cortex or epiretinal membrane $[1,2,9]$, retinal vascular microfolds [2], and posterior staphyloma may be contributing factors $[1,2,8]$. Previous studies focused on the macular area, and few studies have examined changes in the optic disc or peripapillary region [1]. In our clinical original author(s) and the source, provide a link to the Creative Commons licence, and indicate if changes were made. The images or other third party material in this article are included in the article's Creative Commons licence, unless indicated otherwise in a credit line to the material. If material is not included in the article's Creative Commons licence and your intended use is not permitted by statutory regulation or exceeds the permitted use, you will need to obtain permission directly from the copyright holder. To view a copy of this licence, visit http://creativecommons.org/licenses/by/4.0/. The Creative Commons Public Domain Dedication waiver (http://creativeco mmons.org/publicdomain/zero/1.0/) applies to the data made available in this article, unless otherwise stated in a credit line to the data. 
observation, we have found that the optic disc in eyes with MF is usually more oval than in eyes without MF. However, the relationship is not well understood.

Swept-source optical coherence tomography (SS-OCT) is a next-generation form of Fourier-domain optical coherence tomography (OCT) with a central wavelength of $1050 \mathrm{~nm}[10,11]$, characterized by enhanced penetration, reduced signal decay, and a higher scanning rate compared with standard spectral-domain OCT [7, 12, 13]. These properties enable improved visualization of deep ocular structures, especially in axially extended eyes $[7,10,14]$, and thus facilitate research of macular diseases in highly myopic eyes.

The purpose of the present study was to investigate the relationship between an oval optic disc and the occurrence of MF and elucidate the underlying mechanism using SS-OCT.

\section{Methods}

\section{Patients}

Patients with unilateral MF were included in this retrospective observational study. The contralateral eyes were used as a control group to avoid possible systemic bias. All of the eyes underwent complete ophthalmologic examinations at the Eye and ENT Hospital of Fudan University, Shanghai, China, between May 2017 and July 2019, that included assessment of best-corrected visual acuity (BCVA), intraocular pressure, and axial length, slit-lamp biomicroscopy, dilated fundus examination, fundus photography, and OCT. The study was conducted according to the ethical standards of the Declaration of Helsinki and was approved by the Institutional Review
Committee of the Eye and ENT Hospital of Fudan University. Informed consent was provided by all patients.

\section{Fundus photographic measurements}

Fundus photographs centered on the macula and optic disc were obtained using a $45^{\circ}$ digital retinal camera (CR-2, Canon Inc., Tokyo, Japan) through dilated pupils. Good-quality images (pixel pitch reaching $4.37 \mu \mathrm{m}$ ) were used for further analysis. Two patients were excluded due to unacceptable image quality. Two trained ophthalmologists (K.Z. and H.C.) measured the biometric parameters described below using ImageJ software (Version 1.49p, National Institutes of Health, MD, USA) in a blinded manner, in accordance with previously reported protocols. The minimal and maximal optic disc diameters were measured and the ovality index (OI) was calculated as the ratio between the minimal and maximal diameter (Fig. 1A) [3, 10, 15-17]. The disc area was manually outlined based on the margin of the optic disc (defined as the inner edge of Elschnig's ring) [16-19]. The angle of optic disc torsion was identified as the deviation of the maximal disc diameter from the fovea-Bruch's membrane opening (BMO) centroid axis [20,21]. The peripapillary atrophy (PPA) $-\beta / \gamma$ zone, often seen as a peripapillary crescent or halo around the optic disc with a distinct margin [3], was characterized by a visible sclera and an atrophic choroid with large choroidal vessels [3, 21, 22]. The PPA- $\beta$ zone is the region containing Bruch's membrane lacking retinal pigment epithelium (RPE), whereas the PPA- $\gamma$ zone is the region lacking Bruch's membrane and $\operatorname{RPE}[3,15,23]$, but is difficult to detect by fundus photograph grading. The circumferential extent of the
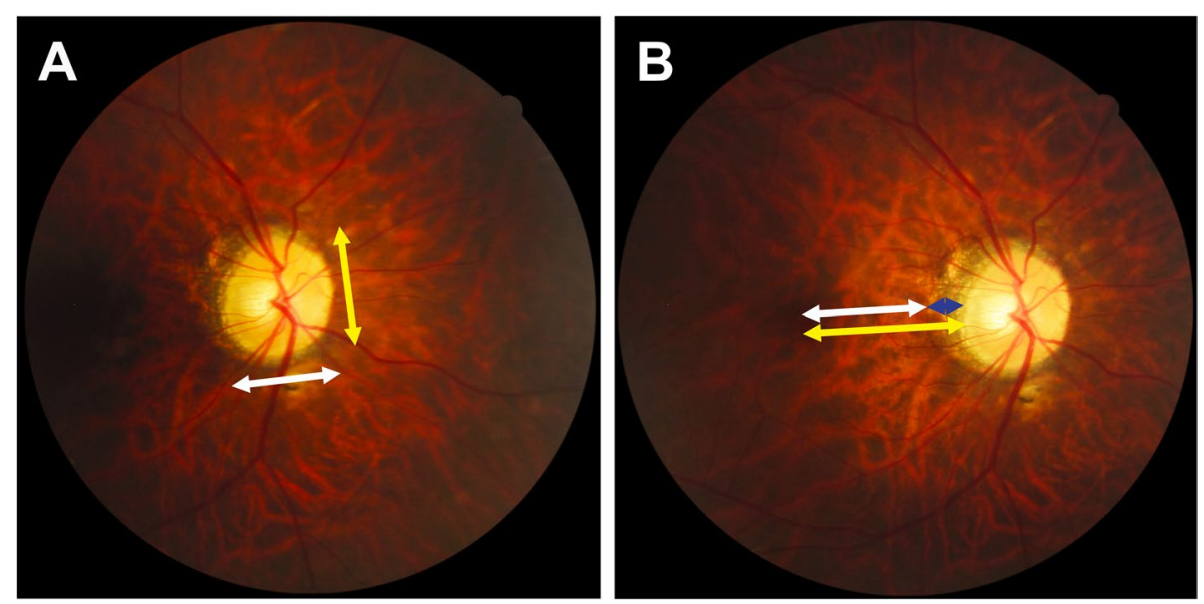

Fig. 1 A schema showing the biometric parameters of the optic disc and PPA- $\beta / Y$ zone measured using fundus photograph. A The ovality index was calculated by dividing the minimal optic disc diameter (white arrow) by the maximal diameter (yellow arrow). B The white arrow indicated the distance between the foveal center and the temporal PPA- $\beta / \gamma$ margin, the yellow arrow indicated the distance between the foveal center and the nasal PPA- $\beta / \gamma$ margin, and the blue arrow indicated the distance between the nasal and temporal PPA- $\beta / \gamma$ margins 

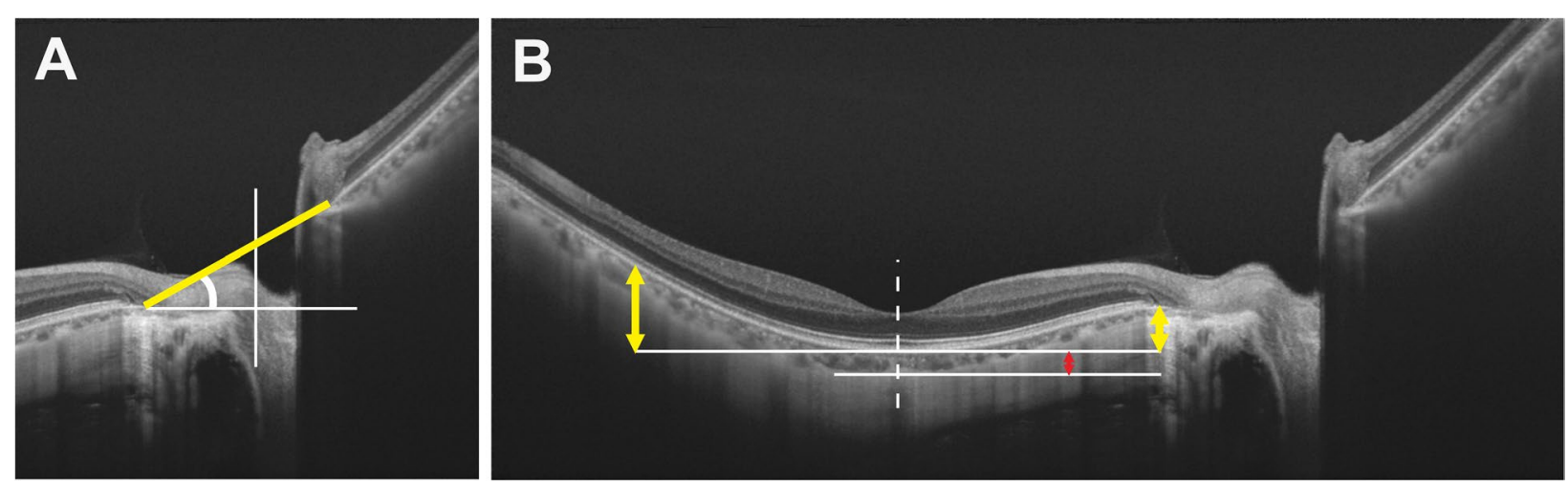

Fig. 2 A schema showing the biometric parameters of the optic disc and posterior staphyloma measured using swept-source optical coherence tomography. A The optic disc tilt was illustrated as the tilt of the line connecting the opening of Bruch's membrane on the nasal and temporal sides of the optic disc (yellow line). B The vertical distances between the anterior edge of the RPE beneath the fovea and the nasal or temporal edges $3 \mathrm{~mm}$ from the fovea (yellow arrow) were averaged as the depth of the posterior staphyloma. The choroidal thickness was defined as the vertical distance between the posterior edge of the Bruch's membrane and chorioscleral interface (red arrow). Dashed line: fovea

PPA- $\beta / \gamma$ zone was recorded as the number of clock hours [23]. The width of the PPA- $\beta / \gamma$ zone was calculated as the distance from the nasal to temporal PPA- $\beta / \gamma$ margins (Fig. 1B) [3].

\section{SS-OCT measurements}

All OCT examinations were performed by one trained ophthalmologist (K.Z.) using a SS-OCT system (DRI OCT - 1 Atlantis, Topcon Corp., Tokyo, Japan) through dilated pupils. The central wavelength of the SS-OCT light source was $1050 \mathrm{~nm}[10,11]$, with an effective axial resolution of $8 \mu \mathrm{m}$ and an axial scan rate of $100,000 \mathrm{~Hz}$ $[10,13]$. Cross-sectional scans centered on the macula and optic disc were obtained along the fovea-BMO axis.
Ninety-six OCT scans were acquired in each image, and were automatically averaged by the built-in software. Good-quality OCT images (signal strength $>60$ ) were used for further analyses. One patient was excluded due to poor image quality. The optic disc tilt was determined as the tilt of the line connecting the opening of Bruch's membrane on the nasal and temporal sides of the optic disc, as previously described (Fig. 2A) [12, 19]. The distance from the foveal center to the peripapillary scleral bending, the distance from the scleral bending to the optic disc edge, and the distance from the foveal center to the optic disc edge were also measured (Fig. 3A) [7]. The angle of scleral bending was defined as the angle between two tangential lines drawn along the surface of the sclera
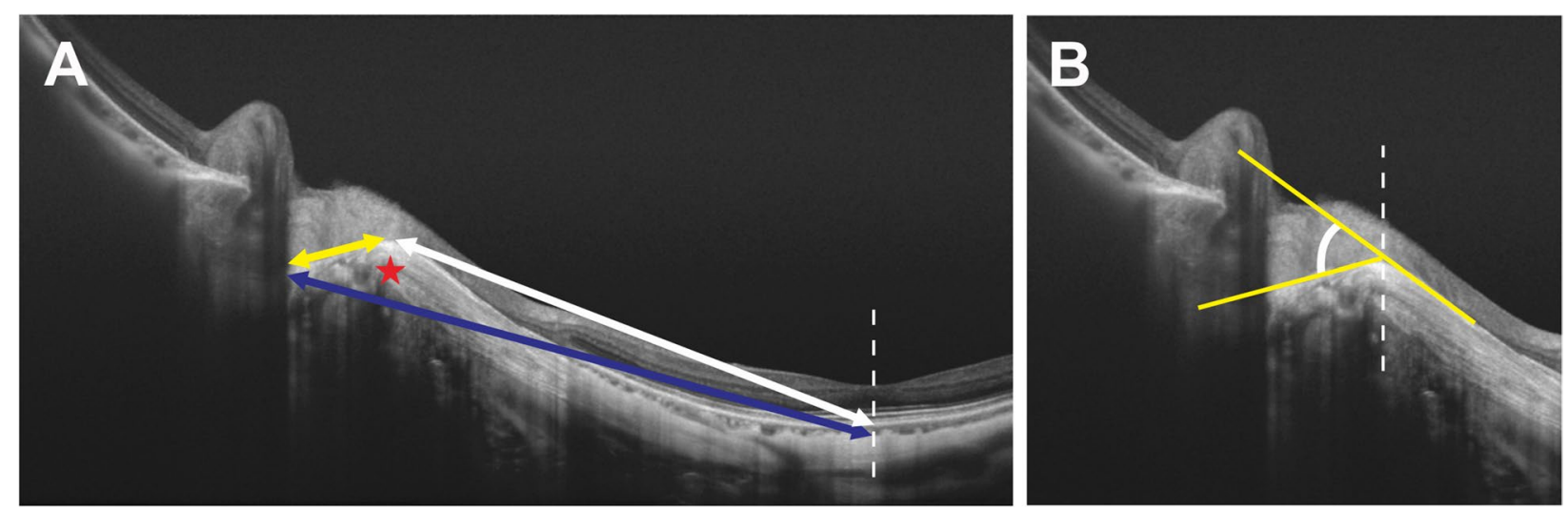

Fig. 3 A schema showing the biometric parameters of peripapillary scleral bending measured using swept-source optical coherence tomography. A The distance from the foveal center to the peripapillary scleral bending (white arrow), the distance from the scleral bending to the optic disc edge (yellow arrow), and the distance from the foveal center to the optic disc edge (blue arrow) were measured. Asterisk: scleral bending; Dashed line: fovea $\mathbf{B}$ The angle of scleral bending was defined as the angle between two tangential lines drawn along the surface of the sclera on both sides of the bending (yellow line) 
on both sides of the bending (Fig. 3B) [7]. The presence and type of posterior staphyloma were determined by identifying the localized outward protrusion of the sclera on the fundus photographs and OCT images according to Ohno-Matsui and Jonas's study $[8,24,25]$. The vertical distances between the anterior edge of the RPE beneath the fovea and the nasal or temporal edges $3 \mathrm{~mm}$ from the fovea were averaged as the depth of the posterior staphyloma (Fig. 2B) [2, 26].

Radial scans centered on the macula were obtained to measure the choroidal thickness (chT). The chT was defined as the vertical distance between the posterior edge of the Bruch's membrane and the chorioscleral interface (Fig. 2B) [13]. Different layers were automatically segmented by the built-in software. The segmentation was carefully checked. If the borderline was misjudged by the software, the correction would be made by two trained ophthalmologists (K.Z. and H.C.). The macular area was divided into three regions, namely, central foveal region $(0.5 \mathrm{~mm}$ from the fovea), parafoveal region $(1.5 \mathrm{~mm}$ from the fovea) and perifoveal region ( $3 \mathrm{~mm}$ from the fovea). The parafoveal and perifoveal regions were divided into four quadrants, namely, the temporal, nasal, inferior and superior quadrants [13]. The averaged chTs of these 9 sectors were obtained for each eye.

\section{Statistical analyses}

Statistical analyses were performed by SPSS software (Version 22.0; SPSS, Inc., Chicago, IL). The BCVA was converted to the logarithm of the minimum angle of resolution (logMAR) for statistical analysis. Student's paired $t$ test was used to compare the ocular and biometric continuous variables. The intra-observer (K.Z.) and inter-observer (K.Z. and H.C.) reproducibility of measurements of the OI, optic disc tilt angle and depth of posterior staphyloma were evaluated by calculating the intraclass correlation coefficients (ICC). Categorical variables were analyzed using McNemar's test or the McNemar-Bowker test. Generalized estimating equation
(GEE) model and linear regression were used to identify associations between clinical factors and the OI. GEE model and logistic regression were performed to determine the factors associated with the occurrence of MF. $P$ values of $<0.05$ were considered statistically significant.

\section{Results}

Fifty eyes of 25 patients with unilateral MF were included in this study, comprising 21 females and four males, with a mean age of $55.88 \pm 11.40$ years. BCVA was not significantly different between the eyes with MF and the contralateral eyes (Table 1). The axial length was $29.43 \pm 1.98 \mathrm{~mm}$ in the eyes with MF and $28.71 \pm 2.62 \mathrm{~mm}$ in the contralateral eyes. The spherical equivalent power was $-13.76 \pm 5.04$ diopters in the eyes with MF and $-12.61 \pm 5.99$ diopters in the contralateral eyes. Six (24\%) eyes showed epiretinal membrane in the eye with MF and 1 (4\%) eye showed epiretinal membrane in the contralateral eyes. The differences were not statistically significant.

\section{Biometric features of the optic disc and PPA- $\beta / \gamma$ zone}

Regarding optic disc characteristics, the minimal disc diameter was significantly smaller $(P=0.050)$, the maximal diameter was greater $(P=0.026)$, and the OI was significantly smaller $(P=0.003)$ in the eyes with MF than in the contralateral eyes (Table 2). The optic disc tilt angle was greater in the eyes with MF $(P=0.023)$, but the optic disc torsion angle was not significantly different between the eyes with MF and the contralateral eyes. The intraobserver reproducibility of the OI $(\mathrm{ICC}=0.921)$ and optic disc tilt angle $(\mathrm{ICC}=0.965)$ was excellent, as was the inter-observer reproducibility $(\mathrm{OI}, \mathrm{ICC}=0.868$; optic disc tilt angle, $I C C=0.940)$. The distance from the foveal center to the temporal PPA- $\beta / \gamma$ margin was significantly shorter $(P=0.015)$ and the PPA- $\beta / \gamma$ zone was wider $(P=0.026)$ in the eyes with MF than in the contralateral eyes.

Table 1 Ocular Characteristics in Patients with Unilateral MF

\begin{tabular}{llll}
\hline Characteristic, $\boldsymbol{n}$ (\%) or Mean \pm SD (Range) & Eyes with MF $(\boldsymbol{n}=\mathbf{2 5})$ & Contralateral Eyes $(\boldsymbol{n}=\mathbf{2 5})$ & $\boldsymbol{P}$ Value \\
\hline BCVA, logMAR & $0.59 \pm 0.46(0.05-2.00)$ & $0.38 \pm 0.47(0.00-2.00)$ & $20 / 48(20 / 20-F C)$ \\
BCVA, Snellen visual acuity ratio & $20 / 78(20 / 22-\mathrm{FC})$ & $16.03 \pm 3.16(9.50-21.00)$ \\
Intraocular pressure, $\mathrm{mmHg}$ & $15.44 \pm 3.37(8.70-21.90)$ & $28.71 \pm 2.62(24.83-34.37)$ & 0.132 \\
Axial length, mm & $29.43 \pm 1.98(25.39-33.94)$ & $-12.61 \pm 5.99(4.50-27.50)$ & 0.165 \\
Spherical equivalent power, diopters & $-13.76 \pm 5.04(5.00-23.00)$ & $24(96)$ & 0.057 \\
High myopia & $24(96)$ & $1(4)$ & 0.242 \\
Epiretinal membrane & $6(24)$ & 0.999 \\
\hline
\end{tabular}

MF Myopic foveoschisis, SD Standard deviation, BCVA Best-corrected visual acuity, logMAR Logarithms of the minimum angle of resolution, FC Finger counting 
Table 2 Biometric Characteristics of the Optic Disc and PPA- $\beta / Y$ Zone of MF

\begin{tabular}{|c|c|c|c|}
\hline Parameter, Mean \pm SD (Range) & Eyes with MF ( $n=25)$ & Contralateral Eyes $(n=25)$ & $P$ Value \\
\hline \multicolumn{4}{|l|}{ Optic disc } \\
\hline Minimal diameter, $\mu \mathrm{m}$ & $1130.11 \pm 408.00(539.14-2161.91)$ & $1259.80 \pm 408.48(515.56-2115.40)$ & 0.050 \\
\hline Maximal diameter, $\mu \mathrm{m}$ & $1916.05 \pm 346.96(1491.79-2798.36)$ & $1795.63 \pm 299.97(1146.18-2345.27)$ & 0.026 \\
\hline $\mathrm{Ol}$ & $0.59 \pm 0.19(0.29-0.99)$ & $0.70 \pm 0.18(0.28-0.97)$ & 0.003 \\
\hline Disc area, $\mathrm{mm}^{2}$ & $2.00 \pm 0.85(0.90-5.16)$ & $2.24 \pm 0.76(1.15-4.70)$ & 0.017 \\
\hline Optic disc tilt angle, degrees & $13.42 \pm 7.30(1.32-26.29)$ & $10.37 \pm 7.28(0.00-23.30)$ & 0.023 \\
\hline Optic disc torsion angle, degrees & $88.86 \pm 10.82(71.70-121.95)$ & $88.52 \pm 7.10(71.51-97.81)$ & 0.893 \\
\hline \multicolumn{4}{|l|}{ PPA- $\beta / \gamma$ zone } \\
\hline Circumferential extent of PPA- $\beta / \gamma$, clock hours & $7.78 \pm 2.75(4.22-12.00)$ & $8.02 \pm 3.25(3.40-12.00)$ & 0.684 \\
\hline Distance from foveal center to optic disc center, $\mu \mathrm{m}$ & $5193.63 \pm 599.73(3873.49-6657.11)$ & $5242.64 \pm 611.52(4212.46-7014.34)$ & 0.739 \\
\hline $\begin{array}{l}\text { Distance from foveal center to temporal PPA- } \beta / \gamma \text { margin, } \\
\mu \mathrm{m}\end{array}$ & $2706.87 \pm 682.09(1444.10-4162.03)$ & $3108.66 \pm 826.24(945.24-5003.61)$ & 0.015 \\
\hline Distance from foveal center to nasal PPA- $\beta / \gamma$ margin, $\mu \mathrm{m}$ & $5114.57 \pm 1224.14(3432.98-8243.46)$ & $5140.42 \pm 1091.65(3275.77-7244.57)$ & 0.870 \\
\hline PPA- $\beta / \gamma$ width, $\mu \mathrm{m}$ & $2407.70 \pm 1540.66(200.30-6302.48)$ & $2031.76 \pm 1490.40(155.50-6126.50)$ & 0.026 \\
\hline
\end{tabular}

MF Myopic foveoschisis, SD Standard deviation, OI Ovality index, PPA- $\beta / \gamma$ Peripapillary atrophy $-\beta / \gamma$

\section{Biometric features of peripapillary scleral bending and posterior staphyloma}

There were no statistically significant differences in the biometric features of the peripapillary scleral bending between the eyes with MF and the contralateral eyes (Table 3). Posterior staphyloma was detected in all the eyes. Based on fundus photography and OCT findings, of the eyes with MF, three (12\%) showed wide macular staphyloma, 20 (80\%) showed narrow macular staphyloma, and two (8\%) showed peripapillary staphyloma, and of the contralateral eyes, 10 (40\%) showed wide macular staphyloma, 13 (52\%) showed narrow macular staphyloma, and two (8\%) showed peripapillary staphyloma. Narrow macular staphyloma was more frequent $(P=0.020)$ and the staphyloma was significantly deeper $(P=0.006)$ in the eyes with MF compared with the contralateral eyes. The ICC of the measurement of depth of posterior staphyloma was 0.959 and 0.929 for intraobserver and inter-observer reproducibility, respectively.

\section{ChTs in the macular area}

The choroid in the central foveal region was significantly thinner in the eyes with MF than in the contralateral eyes $(P=0.008)$ (Supplementary Table S1). A similar trend was found across all sectors. A gradual thinning of the choroid from the perifoveal region toward parafoveal region and from the parafoveal region toward the central foveal region was observed in the macular area.

\section{Associations between clinical factors and the $\mathrm{OI}$}

Simple linear regression revealed that the optic disc tilt angle was negatively correlated with the OI (regression coefficient $[\beta]=-0.017,95 \%$ confidence interval $[\mathrm{CI}]=-0.023$ to $\left.-0.011, P=0.000, r^{2}=0.431\right)$. In GEE

Table 3 Biometric Characteristics of Peripapillary Scleral Bending and Posterior Staphyloma of MF

\begin{tabular}{|c|c|c|c|}
\hline Parameter, Mean \pm SD (Range) & Eyes With MF ( $n=25$ ) & Contralateral Eyes $(n=25)$ & $P$ Value \\
\hline \multicolumn{4}{|l|}{ Peripapillary scleral bending } \\
\hline Distance from foveal center to scleral bending, $\mu$ m & $3862.19 \pm 943.87(1992.22-6513.16)$ & $3961.86 \pm 916.67(2003.24-6624.96)$ & 0.619 \\
\hline Distance from foveal center to optic disc edge, $\mu \mathrm{m}$ & $4721.52 \pm 925.26(2700.75-7295.10)$ & $4630.41 \pm 880.71(3057.59-6419.77)$ & 0.589 \\
\hline Distance from scleral bending to optic disc edge, $\mu \mathrm{m}$ & $910.66 \pm 313.72(249.34-1589.72)$ & $744.82 \pm 470.99(0.00-1759.49)$ & 0.150 \\
\hline Scleral bending angle, degrees & $19.08 \pm 13.23(1.60-55.69)$ & $17.67 \pm 14.02(0.00-52.85)$ & 0.636 \\
\hline \multicolumn{4}{|l|}{ Posterior staphyloma } \\
\hline Staphyloma type & & & 0.020 \\
\hline Type I. wide, macular & $3(12)$ & $10(40)$ & \\
\hline Type II. narrow, macular & $20(80)$ & $13(52)$ & \\
\hline Type III. Peripapillary & $2(8)$ & $2(8)$ & \\
\hline Average staphyloma depth, $\mu \mathrm{m}$ & $652.71 \pm 181.12(340.27-1071.13)$ & $524.37 \pm 215.24(130.44-958.71)$ & 0.006 \\
\hline
\end{tabular}

MF Myopic foveoschisis, SD Standard deviation 
model (multiple linear regression), spherical equivalent refraction $(\beta=-0.016,95 \% \mathrm{CI}=-0.025$ to -0.006 , $P=0.001)$, narrow macular staphyloma $(\beta=-0.152,95 \%$ $\mathrm{CI}=-0.240$ to $-0.065, P=0.001)$ and the occurrence of MF $(\beta=-0.073,95 \% \mathrm{CI}=-0.137$ to $-0.009, P=0.026)$ were the independent factors associated with the OI (Table 4).

\section{Associations between clinical factors and the occurrence of MF}

Univariate logistic regression analysis showed that narrow macular staphyloma (odds ratio $[\mathrm{OR}]=3.692$, 95\% $\mathrm{CI}=1.052-12.957, P=0.041)$ and staphyloma depth $(\mathrm{OR}=1.003,95 \% \mathrm{CI}=1.000-1.006, P=0.037)$ were positively associated with the occurrence of MF (Table 5).

Table 4 GEE Model (Multiple Linear Regression) of Clinical Factors Associated with the OI

\begin{tabular}{llll}
\hline Variables Included in the Model & $\boldsymbol{\beta}$ & $\mathbf{9 5 \%} \mathrm{Cl}$ & $\boldsymbol{P}$ Value \\
\hline Axial length, mm & 0.006 & -0.015 to 0.027 & 0.559 \\
$\begin{array}{l}\text { Spherical equivalent power, } \\
\text { diopters }\end{array}$ & -0.016 & -0.025 to -0.006 & 0.001 \\
Posterior staphyloma & & & \\
$\quad$ Type II. narrow, macular & -0.152 & -0.240 to -0.065 & 0.001 \\
$\quad$ Average staphyloma depth, $\mu \mathrm{m}$ & 0.000 & 0.000 to 0.000 & 0.179 \\
MF & -0.073 & -0.137 to -0.009 & 0.026
\end{tabular}

GEE Generalized estimating equation, OI Ovality index, $\beta$ Regression coefficient, $\mathrm{Cl}$ Confidence interval, MF Myopic foveoschisis
After adjusting for confounding variables, GEE model (multivariate logistic regression) estimated that narrow macular staphyloma $(\mathrm{OR}=3.841,95 \% \mathrm{CI}=1.333-11.066$, $P=0.013)$ was the only independent factor associated with the occurrence of MF.

\section{Discussion}

MF was first reported by Takano and Kishi in 1999 [8], and it affects $8-34 \%$ of highly myopic eyes [9]. The pathogenesis of MF is still unclear. Most studies have focused on the macular area and few have evaluated the changes in the optic disc or peripapillary region [1]. In our clinical observation, the optic disc is more oval in eyes with MF than in eyes without MF. This finding prompted discussions on why the optic disc is more oval in MF, and whether there is a relationship between an oval disc and the occurrence of MF.

The optic disc was markedly stretched in highly myopic eyes but its morphology is difficult to evaluate in a standardized manner [10]. The OI, defined as the ratio between the minimal and maximal optic disc diameters $[3,10,15-17]$, is an objective and easily calculated parameter that can be measured on fundus examination without requiring additional imaging devices [10, 27]. In this study, the OI was significantly smaller in the eyes with MF than in the contralateral eyes. The optic disc tilt angle was significantly greater in the eyes with MF, but the optic disc torsion angle was not significantly different between the eyes with MF and the contralateral eyes. Simple linear regression analysis revealed that the optic

Table 5 Logistic Regression of Clinical Factors Associated with the Occurrence of MF

\begin{tabular}{|c|c|c|c|c|}
\hline \multirow[t]{2}{*}{ Variables Included in the Model } & \multicolumn{2}{|l|}{ Univariate } & \multicolumn{2}{|c|}{ Multivariate (GEE model) } \\
\hline & OR $(95 \% \mathrm{Cl})$ & $P$ Value & OR $(95 \% \mathrm{Cl})$ & $P$ Value \\
\hline \multicolumn{5}{|l|}{ Demographic and ocular characteristics } \\
\hline Age, years & $1.000(0.953-1.050)$ & $>0.999$ & $0.991(0.969-1.013)$ & 0.413 \\
\hline Axial length, mm & $1.143(0.896-1.457)$ & 0.282 & & \\
\hline \multicolumn{5}{|l|}{ Optic disc } \\
\hline $\mathrm{Ol}$ & $0.043(0.002-1.066)$ & 0.055 & & \\
\hline Disc tilt angle, degrees & $1.058(0.979-1.143)$ & 0.152 & & \\
\hline Disc torsion angle, degrees & $1.004(0.945-1.067)$ & 0.896 & & \\
\hline \multicolumn{5}{|l|}{ PPA- $\beta / \gamma$ zone } \\
\hline PPA- $\beta / \gamma$ width, $\mu \mathrm{m}$ & $1.000(1.000-1.001)$ & 0.388 & & \\
\hline \multicolumn{5}{|l|}{ Peripapillary scleral bending } \\
\hline Scleral bending angle, degrees & $1.008(0.967-1.050)$ & 0.716 & & \\
\hline \multicolumn{5}{|l|}{ Posterior staphyloma } \\
\hline Type II. narrow, macular & $3.692(1.052-12.957)$ & 0.041 & $3.841(1.333-11.066)$ & 0.013 \\
\hline Average staphyloma depth, $\mu \mathrm{m}$ & $1.003(1.000-1.006)$ & 0.037 & & \\
\hline Central foveal ChT, $\mu \mathrm{m}$ & $0.978(0.955-1.001)$ & 0.060 & & \\
\hline
\end{tabular}


disc tilt degree was significantly correlated with the OI. These results suggest that the ovality of the optic disc in eyes with MF results from the optic disc tilt around the vertical disc axis [10], in which the temporal disc edge is angled towards the rear of the eye and the nasal edge is angled towards the front of the eye [12].

We investigated the relationship between the optic disc tilt and the occurrence of MF further. Previous studies reported that optic disc tilt may be caused by the increased axial length and the step configuration of the scleral bed $[6,10,28,29]$. The biometric features of the optic disc, PPA- $\beta / \gamma$ zone, peripapillary scleral bending, and posterior staphyloma were determined. GEE model (multiple linear regression) revealed that spherical equivalent refraction, narrow macular staphyloma, and the occurrence of MF were the independent factors associated with the OI. Several other studies have reported similar findings. How et al. [28] and Samarawickrama et al. [6] reported that myopia spherical equivalent was a significant risk factor for the optic disc tilt. However, Tay et al. reported that greater optic disc ovality correlated with longer axial length [17]. Asai et al. reported that the OI showed significant associations with age, macular choroidal thickness, and the depth of staphyloma [10]. In the present study, neither axial length nor depth of staphyloma were associated with the OI, unlike in previous studies $[10,17,28]$. Our results indicate that optic disc tilt is affected by local protrusion in the macular area rather than general global enlargement. In parallel, we investigated the factors associated with the occurrence of MF. The OI was smaller, and the PPA- $\beta / \gamma$ zone was wider, and the choroid in the central foveal region was thinner in the eyes with MF than in the contralateral eyes. Narrow macular staphyloma was more frequent and the staphyloma was deeper in the eyes with MF compared with the contralateral eyes. After adjusting for confounding variables, GEE model (multivariate logistic regression) revealed that narrow macular staphyloma was the only independent factor associated with the occurrence of MF. Overall, these results indicate that the optic disc tilt is likely to be due to local staphyloma protrusion in the macular area, which shows a significant association with the occurrence of MF.

Posterior staphyloma, a circumscribed outpouching of the posterior pole, was classified into six types according to a study by Ohno-Matsui and Jonas [24]. Wide macular, narrow macular, and peripapillary staphyloma were found in our study. Narrow macular staphyloma was more frequent in the eyes with MF than in the contralateral eyes. Narrow macular staphyloma, characterized by a protrusion in the macular area, has a much smaller curvature radius in the fovea than in the adjacent eye wall [24]. A distinct and abrupt change in the scleral curvature was visible at the staphyloma edge. We speculate that during the development of staphyloma, the posterior pole expands in an asymmetrical temporal manner [29], and the optic nerve head is mechanically pulled toward the nasal direction (Fig. 4) [6, 23, 30]. Therefore, the optic disc may tilt over the vertical disc axis. The enlarged PPA- $\beta / \gamma$ zone and the shortened distance from the foveal center to the temporal PPA- $\beta / \gamma$ margin in the eyes with MF suggest that the PPA- $\beta / \gamma$ zone margin has shifted temporally $[3,30]$, supporting our proposed mechanism. Additionally, progressive mechanical stretching of the posterior pole in the macular area resulting from the staphyloma protrusion may increase the outward posterior traction on the neurosensory retina [9], thereby increasing the susceptibility of highly myopic eyes to experience foveoschisis [8]. However, chorioretinal atrophy was more frequent than foveoschisis in eyes with wide macular staphyloma $[1,24,29]$. We speculate that the sclera of the posterior pole protrudes relatively inwardly in this flat-based staphyloma (i.e., from the nasal optic nerve head to the temporal macula) [24]. This protrusion may weaken the outward posterior traction of the staphyloma and the inward tangential traction from the posterior vitreous cortex or the epiretinal membrane [8], and hence reduce the incidence of retinal splitting and protect against the development of MF. In addition, the optic disc may be flatter in eyes with a wide macular staphyloma because the traction force is more isotropic under the optic nerve head in these eyes.

In this study, high-resolution images of the macula and optic disc morphological features were obtained using SS-OCT. The high penetration of the SS-OCT light source and the low signal decay along the optic axis improved the visualization of deep ocular structures [7, $10,14]$. Our study demonstrates that SS-OCT is a useful imaging tool for highly myopic eyes. Using this imaging technique, we also measured the optic disc tilt angle, torsion angle, and other peripapillary features that were not based on the traditional horizontal axis but instead on the fovea-BMO axis. Several recent studies have suggested that it is better to acquire images along the fovea$\mathrm{BMO}$ axis because the horizontal axis may be affected by the disc-fovea angle, and may introduce potential bias $[13,16,31]$.

There are several limitations that should be acknowledged. First, this is a retrospective cross-sectional study. The sequential SS-OCT images should be obtained to observe the changes of optic disc tilt and MF over time. The longitudinal analysis, which may better determine the relationship between the OI and the occurrence of MF, should be performed in future studies. Second, we did not perform three-dimensional magnetic resonance imaging to determine the posterior staphyloma 


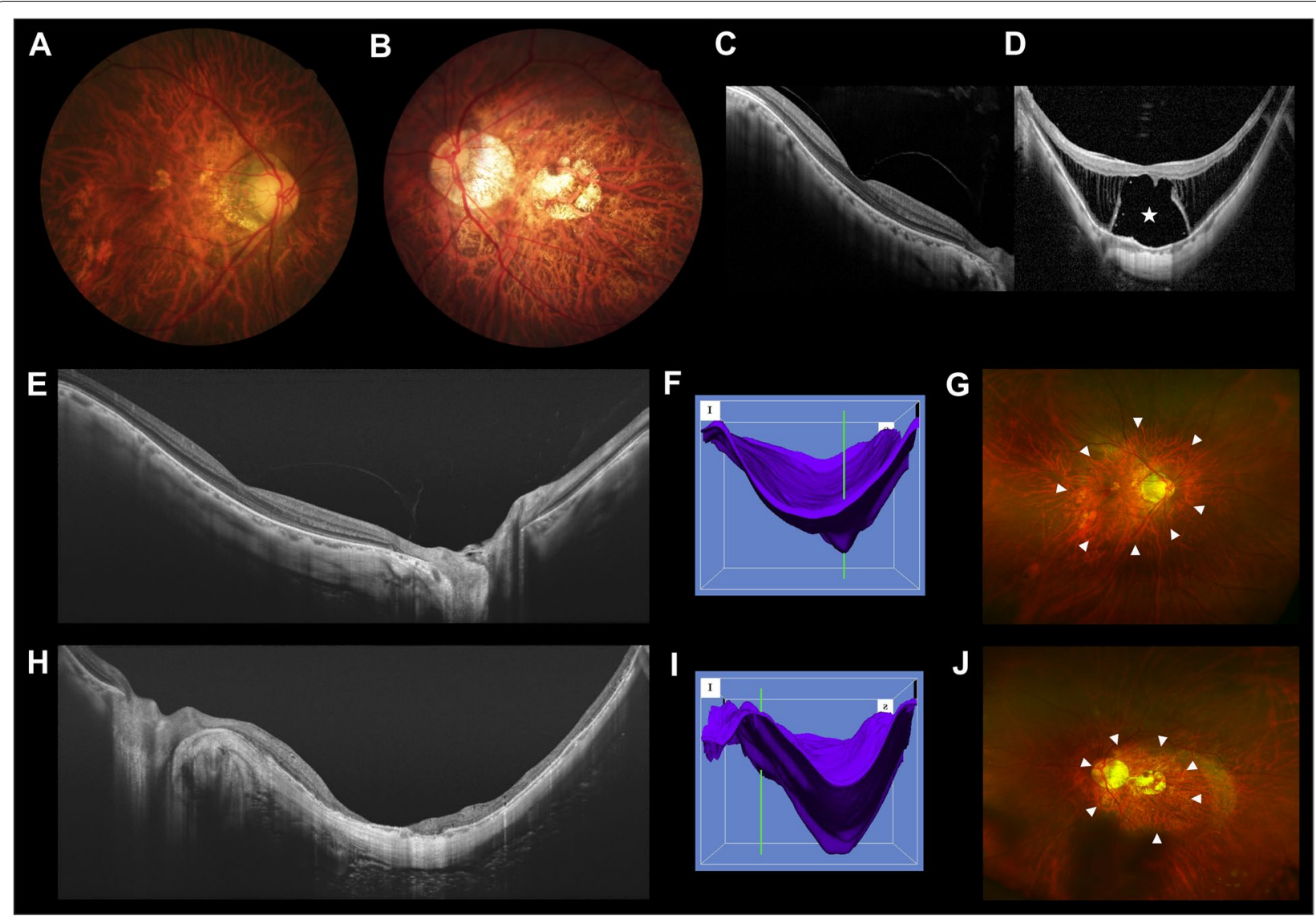

Fig. 4 A 55-year-old female presented with unilateral myopic foveoschisis (MF) (left eye). A,B Fundus photographs showed the optic disc was more oval and the PPA- $\beta / Y$ zone was wider in the eye with MF (B) than in the contralateral eye (A). C,D Preoperative spectral-domain optical coherence tomography (OCT) image showed MF and foveal detachment (asterisk) in the left eye (D), whereas the right eye was unaffected (C). E Postoperative swept-source OCT (SS-OCT) image of the right eye. F Three-dimensional (3D) image of the chorioscleral interface reconstructed by the SS-OCT built-in software showed the scleral outpouching, resulting from a wide macular staphyloma in the right eye. Line: optic nerve head. G The edge of the staphyloma (arrowheads) was visible in the right ultrawide-field fundus photograph. H Postoperative SS-OCT image of the left eye. (I) 3D image showed the scleral outpouching, resulting from a narrow macular staphyloma in the left eye. Line: optic nerve head. JThe edge of the staphyloma (arrowheads) was outlined in the left ultrawide-field fundus photograph

location [5]. Conventional fundus photographs and SSOCT images may not be wide enough to visualize the entire length of the staphylomas, so some staphylomas, mainly peripapillary and nasal staphylomas [24], might not be classified accurately. Third, all of the parameters were measured on two-dimensional images rather than over the real curvature of the eyes [3]. Fourth, the current study comprised a small number of subjects, so the results may not represent the general population of individuals with MF. Therefore, further studies with a larger sample size are necessary.

\section{Conclusions}

This study demonstrated that an oval optic disc in eyes with MF resulted from increased tilt around the vertical disc axis. The optic disc tilt was related to narrow macular staphyloma, which was the only independent factor associated with the occurrence of MF. The study investigated the quantification of an oval optic disc in relation to MF using SS-OCT. The clinical relevance needs further exploration through longitudinal analysis.

\section{Abbreviations}

BMO: Bruch's membrane opening; BCVA: Best-corrected visual acuity; chT: Choroidal thickness; GEE: Generalized estimating equation; ICC: Intraclass correlation coefficients; logMAR: logarithm of the minimum angle of resolution; MF: Myopic foveoschisis; MH: Macular hole; OCT: Optical coherence tomography; Ol: Ovality index; PPA: Peripapillary atrophy; RPE: Retinal pigment epithelium; SS-OCT: Swept-source optical coherence tomography.

\section{Supplementary Information}

The online version contains supplementary material available at https://doi. org/10.1186/s12886-022-02319-9.

Additional file 1. 


\section{Acknowledgements \\ Not applicable.}

\section{Authors' contributions}

KZ analyzed the data and drafted the manuscript. BL, KW, FS and RJ participated in data acquisition. HC, GX and QC reviewed and revised the manuscript. All the authors have read and approved the final manuscript.

\section{Funding}

This work was supported by research grants from the Shanghai Sailing Program (20YF1404800), the Youth Project of the National Natural Science Fund (82000918) and the Xuhui District Health Commission of Shanghai (XHLHGG201807).

\section{Availability of data and materials}

The datasets generated and/or analyzed during the current study available from the corresponding author on reasonable request.

\section{Declarations}

\section{Ethics approval and consent to participate}

All procedures performed in the study involving human participants were approved by the Institutional Review Committee of the Eye and ENT Hospital of Fudan University, and were in accordance with the ethical standards of the 1964 Declaration of Helsinki and its later amendments. Informed consent was obtained from all participants.

\section{Consent for publication}

Not applicable.

\section{Competing interests}

The authors declare that they have no competing interests.

\section{Author details}

'Eye Institute and Department of Ophthalmology, Eye and ENT Hospital, Fudan University, 83 Fen Yang Road, Shanghai 200031, China. ${ }^{2}$ Shanghai Key Laboratory of Visual Impairment and Restoration, Shanghai, China. ${ }^{3} \mathrm{NHC}$ Key Laboratory of Myopia (Fudan University); Key Laboratory of Myopia, Chinese Academy of Medical Sciences, Shanghai, China.

Received: 26 April 2021 Accepted: 17 February 2022

Published online: 22 February 2022

\section{References}

1. Shimada N, Ohno-Matsui K, Nishimuta A, Tokoro T, Mochizuki M. Peripapillary changes detected by optical coherence tomography in eyes with high myopia. Ophthalmology. 2007;114:2070-6.

2. lida Y, Hangai M, Yoshikawa M, Ooto S, Yoshimura N. Local biometric features and visual prognosis after surgery for treatment of myopic foveoschisis. Retina. 2013;33:1179-87.

3. Nonaka A, Hangai M, Akagi T, Mori S, Nukada M, Nakano N, et al. Biometric features of peripapillary atrophy beta in eyes with high myopia. Invest Ophthalmol Vis Sci. 2011;52:6706-13.

4. Holden BA, Fricke TR, Wilson DA, Jong M, Naidoo KS, Sankaridurg P, et al. Global prevalence of myopia and high myopia and temporal trends from 2000 through 2050. Ophthalmology. 2016;123:1036-42.

5. Zhao XJ, Jiang HY, Li YH, Liu BQ, Xu HX, Zhou J, et al. Correlations between the optic nerve head morphology and ocular biometrics in highly myopic eyes. Int J Ophthalmol. 2018;11:997-1001.

6. Samarawickrama C, Mitchell P, Tong L, Gazzard G, Lim L, Wong TY, et al. Myopia-related optic disc and retinal changes in adolescent children from Singapore. Ophthalmology. 2011;118:2050-7.

7. Akagi T, Hangai M, Kimura Y, Ikeda HO, Nonaka A, Matsumoto A, et al. Peripapillary scleral deformation and retinal nerve fiber damage in high myopia assessed with swept-source optical coherence tomography. Am J Ophthalmol. 2013:155:927-36

8. Shinohara K, Tanaka N, Jonas JB, Shimada N, Moriyama M, Yoshida T, et al. Ultrawide-field OCT to investigate relationships between myopic macular retinoschisis and posterior staphyloma. Ophthalmology. 2018;125:1575-86.

9. Kim KS, Lee SB, Lee WK. Vitrectomy and internal limiting membrane peeling with and without gas tamponade for myopic foveoschisis. Am J Ophthalmol. 2012;153:320-6.e1.

10. Asai T, Ikuno Y, Akiba M, Kikawa T, Usui S, Nishida K. Analysis of peripapillary geometric characters in high myopia using swept-source optical coherence tomography. Invest Ophthalmol Vis Sci. 2016;57:137-44.

11. Potsaid B, Baumann B, Huang D, Barry S, Cable AE, Schuman JS, et al. Ultrahigh speed 1050nm swept source/Fourier domain OCT retinal and anterior segment imaging at 100,000 to 400,000 axial scans per second. Opt Express. 2010;18:20029-48.

12. Pan T, Su Y, Yuan ST, Lu HC, Hu ZZ, Liu QH. Optic disc and peripapillary changes by optic coherence tomography in high myopia. Int J Ophthalmol. 2018;11:874-80.

13. Deng J, Li X, Jin J, Zhang B, Zhu J, Zou H, et al. Distribution pattern of choroidal thickness at the posterior pole in chinese children with myopia. Invest Ophthalmol Vis Sci. 2018;59:1577-86.

14. Ohno-Matsui K, Akiba M, Moriyama M, Ishibashi T, Tokoro T, Spaide RF. Imaging retrobulbar subarachnoid space around optic nerve by sweptsource optical coherence tomography in eyes with pathologic myopia. Invest Ophthalmol Vis Sci. 2011;52:9644-50.

15. Dai Y, Jonas JB, Huang H, Wang M, Sun X. Microstructure of parapapillary atrophy: beta zone and gamma zone. Invest Ophthalmol Vis Sci. 2013:54:2013-8.

16. Qiu K, Wang G, Lu X, Zhang R, Sun L, Zhang M. Application of the ISNT rules on retinal nerve fibre layer thickness and neuroretinal rim area in healthy myopic eyes. Acta Ophthalmol. 2018;96:161-7.

17. Tay E, Seah SK, Chan SP, Lim AT, Chew SJ, Foster PJ, et al. Optic disk ovality as an index of tilt and its relationship to myopia and perimetry. Am J Ophthalmol. 2005:139:247-52.

18. Samarawickrama C, Pai A, Huynh SC, Burlutsky G, Jonas JB, Mitchell P. Measurement of optic nerve head parameters: comparison of optical coherence tomography with digital planimetry. J Glaucoma. 2009:18:571-5.

19. Hwang YH, Yoo C, Kim YY. Myopic optic disc tilt and the characteristics of peripapillary retinal nerve fiber layer thickness measured by spectral-domain optical coherence tomography. J Glaucoma. 2012;21:260-5

20. Park HY, Lee K, Park CK. Optic disc torsion direction predicts the location of glaucomatous damage in normal-tension glaucoma patients with myopia. Ophthalmology. 2012:119:1844-51.

21. Li Z, Guo X, Xiao O, Lee PY, Liu R, Wang D, et al. Optic disc features in highly myopic eyes: the ZOC-BHVI high myopia cohort study. Optom Vis Sci. 2018;95:318-22

22. Jonas JB, Nguyen XN, Gusek GC, Naumann GO. Parapapillary chorioretinal atrophy in normal and glaucoma eyes. I. Morphometric data. Invest Ophthalmol Vis Sci. 1989;30:908-18.

23. Dai $Y$, Jonas JB, Ling Z, Wang X, Sun X. Unilateral peripapillary intrachoroidal cavitation and optic disk rotation. Retina. 2015;35:655-9.

24. Ohno-Matsui K, Jonas JB. Posterior staphyloma in pathologic myopia. Prog Retin Eye Res. 2019;70:99-109.

25. Shinohara K, Moriyama M, Shimada N, Yoshida T, Ohno-Matsui K. Characteristics of peripapillary staphylomas associated with high myopia determined by swept-source optical coherence tomography. Am J Ophthalmol. 2016;169:138-44.

26. Ikuno $Y$, Tano $Y$. Retinal and choroidal biometry in highly myopic eyes with spectral-domain optical coherence tomography. Invest Ophthalmol Vis Sci. 2009;50:3876-80.

27. Takasaki H, Higashide T, Takeda H, Ohkubo S, Sugiyama K. Relationship between optic disc ovality and horizontal disc tilt in normal young subjects. Jpn J Ophthalmol. 2013;57:34-40.

28. How AC, Tan GS, Chan YH, Wong TT, Seah SK, Foster PJ, et al. Population prevalence of tilted and torted optic discs among an adult Chinese population in Singapore: the Tanjong Pagar study. Arch Ophthalmol. 2009:127:894-9.

29. Vongphanit J, Mitchell P, Wang JJ. Prevalence and progression of myopic retinopathy in an older population. Ophthalmology. 2002;109:704-11. 
30. Kim TW, Kim M, Weinreb RN, Woo SJ, Park KH, Hwang JM. Optic disc change with incipient myopia of childhood. Ophthalmology. 2012;119:21-6.e1-3.

31. Ghassabi Z, Nguyen AH, Amini N, Henry S, Caprioli J, Nouri-Mahdavi K. The fovea-BMO axis angle and macular thickness vertical asymmetry across the temporal raphe. J Glaucoma. 2018;27:993-8.

\section{Publisher's Note}

Springer Nature remains neutral with regard to jurisdictional claims in published maps and institutional affiliations.

- fast, convenient online submission

- thorough peer review by experienced researchers in your field

- rapid publication on acceptance

- support for research data, including large and complex data types

- gold Open Access which fosters wider collaboration and increased citations

- maximum visibility for your research: over $100 \mathrm{M}$ website views per year

At $\mathrm{BMC}$, research is always in progress.

Learn more biomedcentral.com/submissions 